\title{
Liste des tableaux
}

Tableau i : Principales phases suivies pour la rédaction du guide (selon la méthodologie du consensus formalisé)

Tableau 0 : Synthèse des différences entre le métier de PSRPM

et la fonction de PCR

Tableau 1.1 : Quelques points marquants de l'évolution de la Physique Médicale en France entre 1953 et 2007

Tableau 1.2 : Obligations réglementaires de recours aux services

des physiciens médicaux

Tableau 1.3 : Renseignements à fournir à l'ASN pour les différents types

d'installations, lors des déclarations et demandes d'autorisation

Tableau 1.4 : Convention utilisée pour la numérotation des articles du code de la santé publique (et des autres codes)

Tableau 1.5 : Numérotation des articles du code de la santé publique qui concernent plus directement les physiciens médicaux

Tableau 1.6 : Principales exigences réglementaires intervenant dans l'exercice professionnel des physiciens médicaux

Tableau 1.7 : Sélection, parmi les critères INCa d'autorisation pour la radiothérapie externe, de ceux qui sont le plus directement liés aux activités et responsabilités du physicien médical 
Tableau 1.8 : Tableau synthétique des principales missions des physiciens médicaux en tant que spécialistes de la physique et de la métrologie des rayonnements

Tableau 1.9: Ordres de grandeur des doses délivrées et ordres de grandeur des incertitudes considérées comme acceptables en fonction du domaine d'application médicale des rayonnements ionisants

Tableau 1.10 : Grandeurs utilisées en France pour fixer les niveaux de référence diagnostiques

Tableau 1.11 : Étapes conduisant à la détermination de la dose en radiothérapie externe et incertitudes standard relatives estimées $(\mathrm{k}=1)$ pour les faisceaux de photons de haute énergie

Tableau 1.12 : Exemples d'éléments influençant la qualité de l'image

Tableau 1.13 : Principaux critères physiques permettant d'apprécier les performances des systèmes d'imagerie

Tableau 1.14 : Liste des principales formations dans lesquelles le physicien médical devrait être impliqué

Tableau 1.15 : Exemples de thèmes de recherche dans lesquels les physiciens médicaux sont impliqués

Tableaux 1.16 a,b,c : Référentiel de bonne conduite professionnelle pour les physiciens exerçant dans un centre de soins

Tableau 1.17 : Référentiel de bonne conduite professionnelle pour les activités de recherche des physiciens médicaux

Tableau 1.18 : Référentiel de bonne conduite professionnelle pour les activités d'enseignement et de formation des physiciens médicaux

Tableau 1.19 : Référentiel de bonne conduite professionnelle pour les résidents de physique médicale

Tableau 1.20 : Référentiel de bonne conduite professionnelle pour les physiciens médicaux exerçant en milieu industriel ou au sein d'une société de service

Tableau 2.1 : Interprétation des 8 principes de base de la norme ISO 9001 dans le contexte d'une structure de physique médicale

Tableau 2.2: Les différentes approches de la sécurité

Tableau 2.3 : Critères de certification HAS les plus pertinents pour les activités de physique médicale

Tableau 3.1 : Avantages et inconvénients des différentes formes de registres

Tableau 3.2 : Principaux registres utilisés dans le cadre des activités de physique médicale

Tableau 3.3 : Règles générales pour l'archivage des données relatives aux équipements et aux patients

Tableau 3.4 : Données relatives à la physique médicale. Archivage et partage des responsabilités

Tableau 3.5 : Exemples d'indicateurs relatifs à l'activité de physique médicale

Tableau 3.6 : Interprétation des écarts de dose significatifs donnant lieu à déclaration des événements significatifs de radioprotection impliquant des patients 
Tableau 3.7 : Conduite à tenir en cas d'incident (ou d'accident) en rapport avec le domaine d'activités du physicien médical

Tableau 3.8 : Principales étapes du processus d'acquisition d'un équipement médical

Tableau 3.9 : Bonnes pratiques pour la mesure de la dose (en RT et RD)

Tableau 3.10 : Bonnes pratiques pour la mesure de l'activité (en Cth et $\mathrm{MN}$ )

Tableau 3.11 : Bonnes pratiques lors de la mise en service d'un TPS

Tableau 3.12 : Recommandations pour la mise en service (et l'utilisation) d'un système d'enregistrement et de contrôle des paramètres de traitement

Tableau 3.13 : Grandes lignes du processus de réception d'un équipement

Tableau 3.14 : Principales étapes du processus de mise en service

d'un équipement

Tableau 3.15 : Caractéristiques générales des Contrôles de Qualité règlementaires

Tableau 3.16: Conduite à tenir en cas de dégradation de la qualité d'image

Tableau 3.17 : Principales étapes de l'introduction d'un changement ou d'une innovation dans une pratique liée à l'utilisation médicale des rayonnements

Tableau A.1 : Exemple de schéma de classement pour les documents relatifs aux activités de physique médicale 


\section{KIVSDJHIQWQWRQDQ $\square$ OTWEOQN}

